\title{
Bone Regeneration of Hydroxyapatite with Granular Form or Porous Scaffold in Canine Alveolar Sockets
}

\author{
SEOK JIN JANG ${ }^{1 *}$, SE EUN KIM ${ }^{2 *}$, TAE SUNG HAN ${ }^{1}$, JUN SIK SON ${ }^{3}$, \\ SEONG SOO $\mathrm{KANG}^{2}$ and SEOK HWA CHOI ${ }^{1}$ \\ ${ }^{1}$ Department of Veterinary Surgery, Veterinary Medical Center and College of Veterinary Medicine, \\ Chungbuk National University, Cheongju, Republic of Korea; \\ ${ }^{2}$ College of Veterinary Medicine, Chonnam National University, Gwangju, Republic of Korea; \\ ${ }^{3}$ High-Tech Fiber R\&D Headquarters, Korea Textile Development Institute, Daegu, Republic of Korea
}

\begin{abstract}
This study was undertaken to assess bone regeneration using hydroxyapatite (HA). The primary focus was comparison of bone regeneration between granular $H A(g H A)$ forms and porous HA ( $p H A)$ scaffold. The extracted canine alveolar sockets were divided with three groups: control, gHA and $p H A$. Osteogenic effect in the gHA and pHA groups showed bone-specific surface and bone mineral density to be significantly higher than that of the control group $(p<0.01)$. Bone volume fraction, bone mineral density, and amount of connective tissue related to disturbing osseointegration of the gHA group was higher than in the pHA group. Quantity of new bone formation of the pHA group was higher than that of the gHA group. This study demonstrated that gHA and pHA are potentially good bone substitutes for alveolar socket healing. For new bone formation during 8 weeks' post-implantation, $H A$ with porous scaffold was superior to the granular form of $H A$.
\end{abstract}

Hydroxyapatite (HA) is an alloplastic material commonly used as a bone graft substitute due to inherent bioactive properties that support osteo-conduction. However, a weakness of HA-based biomaterials is their slow degradation and absorption in vivo. The granulated form of calcium phosphate ceramics has a regenerative effect within an alveolar bone defect (1). HA is highly crystalline because of the sintering process and has a larger particle size than bone

This article is freely accessible online.

*These Authors contributed equally to this study.

Correspondence to: Seok Hwa Choi, DVM, Ph.D., Professor, Department of Veterinary Surgery, Veterinary Medical Center and College of Veterinary Medicine, Chungbuk National University, Cheongju, 28644, Republic of Korea. E-mail: shchoi@cbnu.ac.kr

Key Words: Alveolar socket, bone regeneration, hydroxyapatite, dog. apatite due to grain growth. These large particles are highly resistant to biodegradation in the body, their osteo-conduction is very low, and they cannot be degraded by osteoclasts (2). The porous HA (pHA) scaffold has good bone regeneration in in vivo performance (3-5). This form of material requires proper size and three-dimensional porosity for demonstrating its intrinsic osteo-conductivity and osteogenic potential within limited and exiguous sites such as dental sockets. An HA scaffold with interconnected pore $300 \mu \mathrm{m}$ in diameter has the most appropriate osteo-conductive capacity (6). Therefore, we hypothesized that HA types and crystallinity influence the rate of bone healing around alveolar sockets.

Bone-filling materials undergo radiological evaluation through determination of the bone mineral density (BMD) and information about bone change. Quantitative radiographic evaluation using $\mathrm{x}$-rays is used to assess bone structure and mechanical properties indirectly. In particular, quantitative computed tomography (CT), and peripheral CT are used in humans for monitoring and treatment of metabolic diseases, skeletal status, and osteoporosis $(7,8)$. Micro-CT yields more information about bone mass and microstructure, and is used for studies of bone metabolism in animal models (9-11). Bone formation and mineralization will affect bone strength; mechanical stability depends on the amount and density of bone (12).

The bone-regeneration ability of each bone substitute is evaluated compared to bone metabolic disorders model by BMD, percentage bone volume, and other bone structural parameters. Our study evaluated bone substitutes in the alveolar socket of mandibles of beagle dogs to determine BMD levels by $\mathrm{CT}$ and bone parameter values by micro-CT at different time points.

In this study, osteogenic potential was compared, focusing on alveolar socket healing and restoration using granular $\mathrm{HA}(\mathrm{gHA})$ forms and porous HA (pHA) scaffolds. Tissue engineering methods were assessed by manufacturing functional, aesthetic HAs that would not affect adjacent tooth structures. 

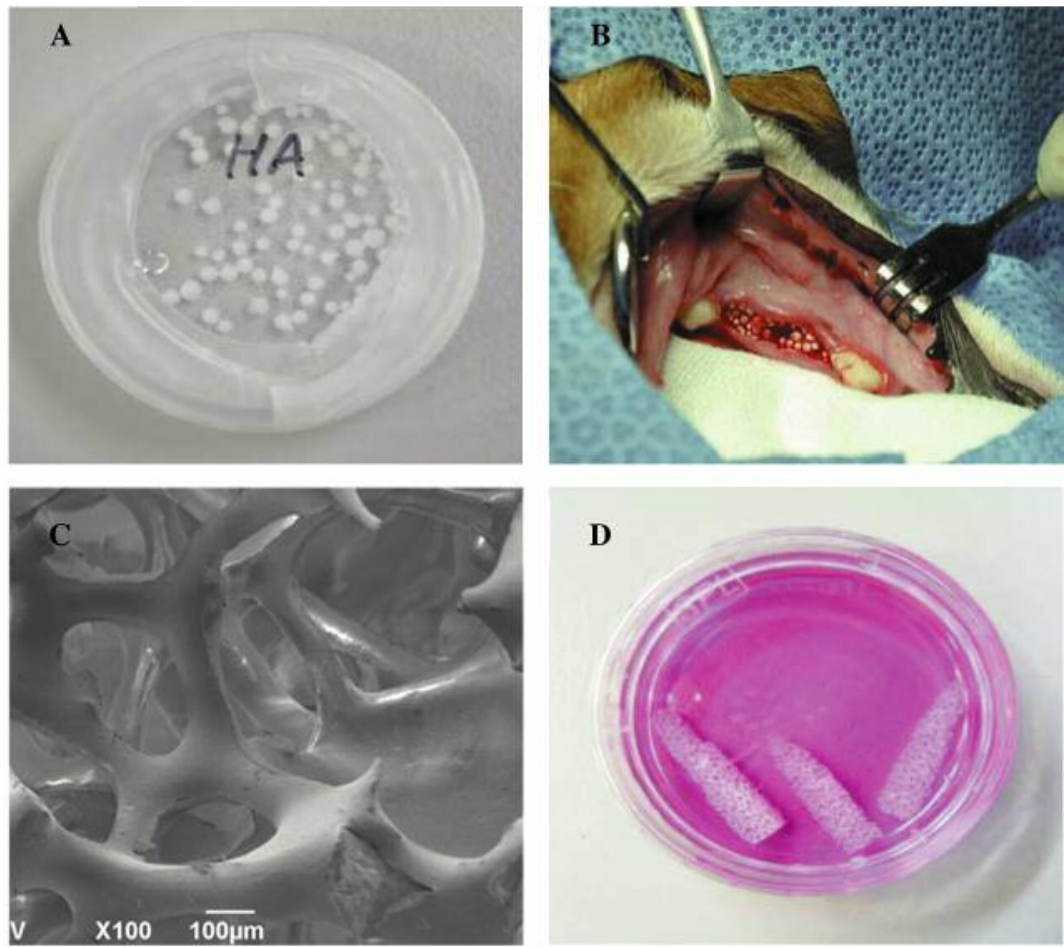

Figure 1. Photographs of granular hydroxyapatite before implantation (A) and in alveolar socket post-implantation (B). Scanning electron microscope image of porous $H A(p H A)$ scaffold $(C)$ and photograph of aseptically prepared pHA before implantation $(D)$.

\section{Materials and Methods}

Experimental animals. Six adult (18-22 months old) male beagle dogs, weighing between 8.5 and $9.5 \mathrm{~kg}$, were included in this study. The protocol for the animal experiment was approved by the Institutional Animal Care and Use Committee of Chungbuk National University (no. 677-14-01). All dogs were kept in individual cages throughout the experimental period. Water and food were supplied ad libitum during the experimental period. The alveolar extraction sites were divided randomly and a split-mouth design was established in three groups: control group: extracted socket only, four alveolar sockets, gHA group: filled with granulated HA, four alveolar sockets, and pHA group: filled with porous HA scaffold, four alveolar sockets.

Preparation of gHA form and pHA scaffold. A gHA slurry was made by mixing $3 \mathrm{~g}$ nano-HA powders (OssGen Co., Daegu, Korea) and binders (2\% high molecular weight polyvinyl alcohol, $1 \%$ carboxymethylcellulose). The gHA slurry was dripped in liquid nitrogen using a micropipette to make a granular structure. After lyophilization, gHA granules were sintered at $1,230^{\circ} \mathrm{C}$ for $3 \mathrm{~h}$. The Particle size of gHA was $500 \mu \mathrm{m}$ to $2 \mathrm{~mm}$.

pHA scaffold was fabricated using a polymeric template-coating technique, as described elsewhere (13). A polyurethane sponge (60 pores per inch) was coated with nano-alumina powders (OssGen Co., Daegu, Korea) in distilled water-based slurry. Binders (3\% high molecular weight polyvinyl alcohol, $3 \%$ carboxymethylcellulose, $5 \%$ ammonium polyacrylate dispersant, and 7\% N,N-dimethylformamide drying agent) were added to the slurry mixture to improve sintering and stabilize the scaffold structure. Coated sponges were dried overnight at room temperature before sintering at $1,500^{\circ} \mathrm{C}$ for $3 \mathrm{~h}$. Final mandible molar root-like shape of pHA scaffold dimensions were $4 \mathrm{~mm}$ in diameter, and $10 \mathrm{~mm}$ in length (Figure 1). The properties of the scaffold produced were observed using a stereoscope (Fisher Micromaster; Fisher Scientific, Fremont, CA, USA) and scanning electron microscope (SEM; EVO 40; ZEISS, Dublin, CA, USA).

Each type of HA was placed in a 24-well petri-dish using plastic forceps. Two milliliters of $70 \%$ ethanol was poured on the well. After $1 \mathrm{~h}$, the ethanol was removed using suction. HA was washed three times with phosphate-buffered saline ( $\mathrm{pH}$ 7.4), soaked in fetal bovine serum-free medium, then left overnight under ultraviolet light. HA was washed three times with fresh medium. After removing the water from the HA, it was implanted.

Surgical procedures. Surgical procedures were performed under sterile conditions and general anesthesia. The left and right mandibular first molars were extracted without injury using the closed extraction technique. The graft site was closed with 4-0 single interrupted absorbable sutures (Surgifit, Ailee Co., Busan, Korea) with gingival flap technique. Enough tissue was elevated for the flap to be placed over the socket without spontaneous retraction. Commercial dog feed soaked in water was fed to minimize the damage of surgical area during the entire experimental period. Antibiotics and analgesics were administered in the postoperative period. 
Evaluation of CT and micro-CT. CT images were taken sequentially at baseline, four, and eight weeks post-implantation to assess the new bone formation and mineralization. CT images were obtained by a single-slice spiral CT (Hi Speed CT/e, GE Medical Co., USA). CT exposures were performed at $120 \mathrm{kVp}(150 \mathrm{~mA})$, with $1 \mathrm{~mm}$ thickness and $512 \times 512$ voxel matrix.

At 8 weeks post-implantation, the harvested mandible was scanned using a micro-CT. A Skyscan Desktop Micro-CT 1172 (Skyscan; Aartselaar, Belgium), with a source voltage of $60 \mathrm{kV}$, a current of $167 \mu \mathrm{A}$, resolution $26.7 \mu \mathrm{m}$ was used to acquire $\mathrm{x}$-ray radiographs. The specimens were attached to a stage that rotated $180^{\circ}$ with images acquired every $0.6^{\circ}$. After scanning, cross-sectional slices were reconstructed and each scan result was reconstructed using the 0.008 0.031 threshold values to distinguish bone and air. The parameters of bone mass and microarchitecture were evaluated using the micro-CT built-in software, including BMD, bone volume, tissue volume, bone surface, percent bone volume, bone-specific surface, bone surface density, trabecular thickness, trabecular number, trabecular separation and interception surface.

Fluorescent bone labeling and preparation of specimens for fluorescence microscopy. To label the mineralized tissue and assess the time course of new bone formation and mineralization, tissue was conducted by a polychrome sequential fluorescent labeling method. Oxytetracycline $(25 \mathrm{mg} / \mathrm{kg}$; Green Cross, Yongin, Gyeonggi, Korea) and calcein $(20 \mathrm{mg} / \mathrm{kg}$, Sigma-Aldrich Co., St Louis, MO, USA) were intravenously injected at four and six weeks after the implantation. Alizarin red S (30 mg/kg, Sigma-Aldrich Co.) was intraperitoneally injected at 8 weeks after the implantation.

The formalin-fixed samples from the sacrificed alveolar sockets in each beagle were dehydrated in a graded series of ethanol, then immersed and infiltrated using Technovit 7200VLC (Technovit; Hatfield, PA, USA) before light catalysis for $24 \mathrm{~h}$. A macrocutting and grinding system (Exakt 310CP series; Exakt Apparatebau, Norderstedt, Germany) was used to produce undecalcified cut and ground sections. A final thickness of approximately $50 \mu \mathrm{m}$ of each section was produced for fluorescence microscopy. Labeling of new bone formation was evaluated with confocal laser scanning microscope. Active bone formation was evaluated relative to the presence or absence, intensity, and width of the fluorochrome markers.

Statistical analysis. Statistical analyses were performed using SPSS statistical software package version 19.0.1.1. (IBM SPSS Statistics for Windows, Version 19.0; IBM Corp., Armonk, NY, USA). Data are presented as the mean \pm standard deviation (SD). Normality and homogeneity of the data were confirmed before analysis of variance (ANOVA). Differences among the experimental groups were assessed by one-way ANOVA followed by Duncan's multiple range tests. Null hypotheses of no difference were rejected if $p$-values were less than 0.05 .

\section{Results}

Material characterization. The particle size of gHA ranged from $500 \mu \mathrm{m}$ to $2 \mathrm{~mm}$ (Figure 1A). Scaffolds with pHA were fabricated using the polymeric sponge replication method, which has received particular attention because it can provide very high porosity with good interconnections between the pores. Open channels were observed to be arranged with isotropic geometry. The stereoscopic image of pHA scaffold is shown in Figure 1C and D.

Findings of quantitative CT. All dogs survived during the experimental period, and all of their wounds healed well. At 8 weeks post-implantation, BMD values were $0.57 \pm 0.15 \mathrm{~g} / \mathrm{cm}^{3}$ and $0.24 \pm 0.11 \mathrm{~g} / \mathrm{cm}^{3}$ for the gHA and pHA groups (Figure 2). The percentage bone volume was $61.72 \pm 6.54 \%$ and $49.58 \pm 13.61 \%$ for gHA and pHA groups (Figure 3). The percentage bone volume of the pHA group was significantly lower than that of the gHA group $(p<0.05)$. The percentage bone volume and BMD were significantly higher in the gHA and the pHA groups than in the control group $(p<0.01)$. BMD values in the gHA group were significantly higher than those of the pHA group at 8 weeks post-implantation $(p<0.05)$.

Findings of micro-CT. New bone formation at the interface between the graft material and the host bone was observed in gHA and pHA groups on micro-CT. There was no alveolar crest resorption in the pHA and gHA groups. The particles of gHA were close to the socket wall on micro-CT images (Figure 4). The granulated HAs were radiopaque due to intrinsic properties. There was no peripheral alveolar bone regression and bone resorption in the gHA and pHA groups. However, a radiolucent zone was observed around the scaffold and the apical area of alveolar socket in the pHA group (Figure 5).

The values of the interception surface were positively correlated with BMD values, and bone-specific surface values were negatively correlated with BMD values (Table I). The trabecular thickness of gHA group was significantly higher than that of pHA group $(p<0.05)$. Trabecular separation of the gHA group was significantly lower than that of the pHA group $(p<0.05)$.

Fluorescence microscopic evaluation. In histological evaluation, the alveolar socket in the gHA and pHA groups were filled with fibrous connective tissues, and new bone formation was seen at the extracted region (Figure 6). It was observed that at 4 and 8 weeks post-implantation, the pHA group had a superior osteogenic effect compared with the gHA group.

The gHA and pHA groups showed very new bone formation around and along the implants. The obtained images are labeled in green and red colors to delineate regions of calcium precipitation labeled by fluorochromes at different times of tissue mineralization. The calcein labeling (green) represents the regions where calcium precipitated at 6 weeks after implantation. The alizarin labeling (red) represents the regions where calcium precipitated at 8 weeks after implantation. Most of the HA at six weeks after implantation was closest to the HA surface in alveolar sockets, and the HA at 8 weeks following implantation were further away from the implant surface. 


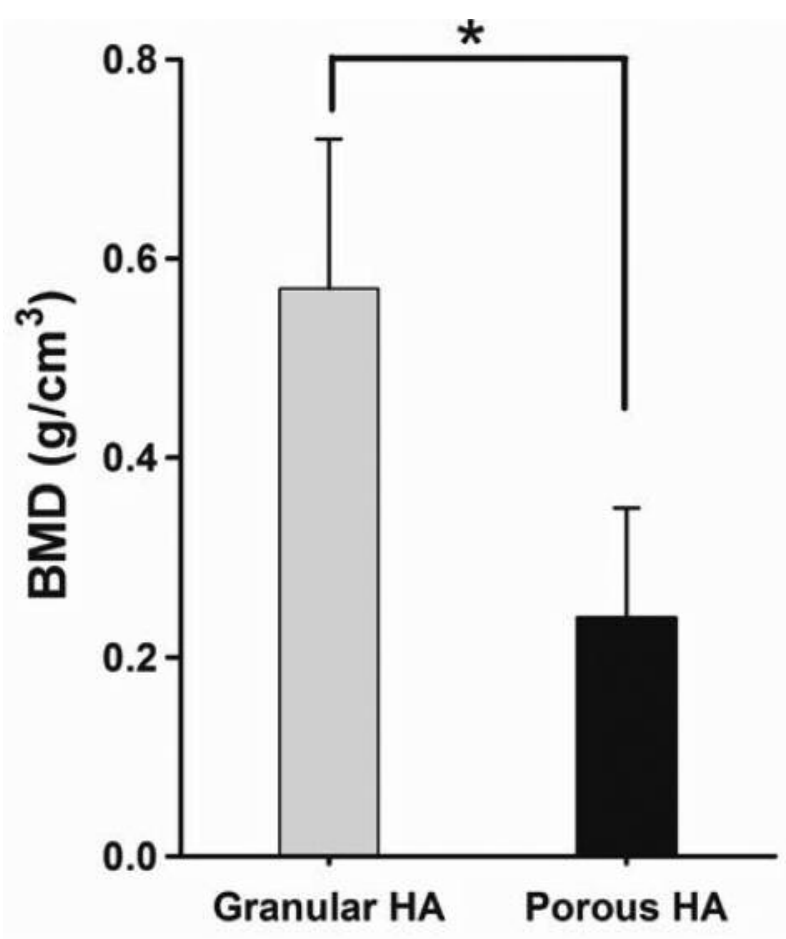

Figure 2. Levels of bone mineral density (BMD) in canine alveolar cavity at 8 weeks post-implantation. Mean $\pm S D(n=4), * p<0.05$.

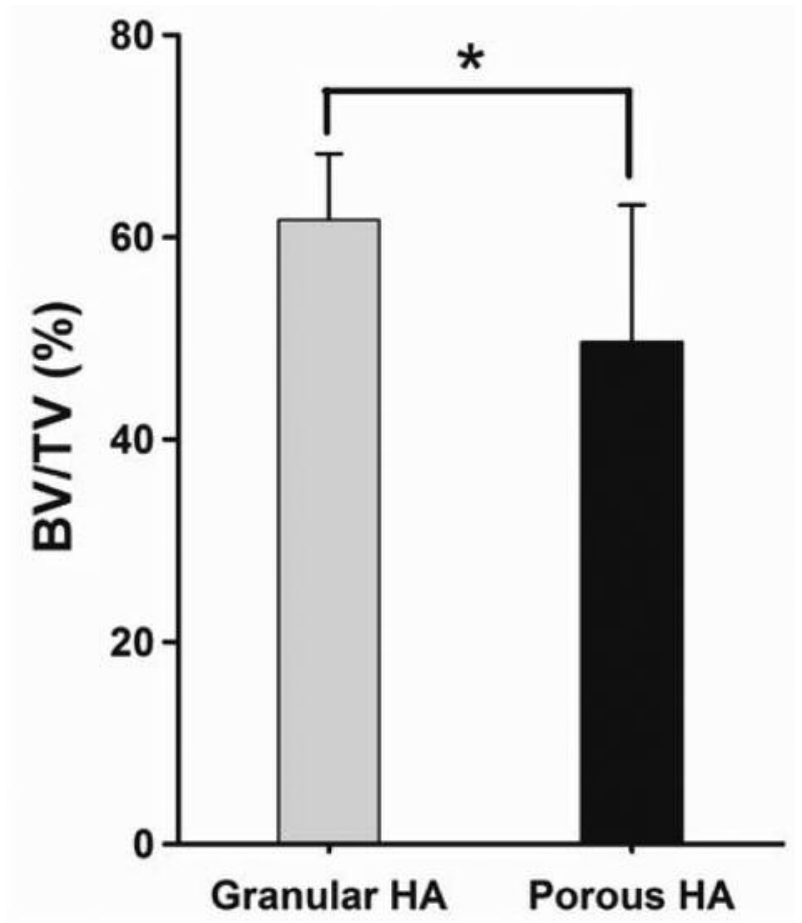

Figure 3. Percentages of bone volume (BV)/tissue volume (TV) in canine alveolar cavity at 8 weeks post-implantation. Mean $\pm S D(n=4),{ }^{*} p<0.05$.

Table I. Bone parameters of canine alveolar sockets at 8 weeks post-implantation of hydroxyapatite. Data are the mean \pm SD $(n=4)$.

\begin{tabular}{lccccc}
\hline Hydroxyapatite & i.S $\left(\mathrm{mm}^{2}\right)$ & $\mathrm{BS} / \mathrm{BV}\left(\mathrm{mm}^{-1}\right)$ & $\mathrm{BS} / \mathrm{TV}\left(\mathrm{mm}^{-1}\right)$ & Tb.Th $(\mathrm{mm})$ & $\left.\mathrm{Tb} . \mathrm{N}(\mathrm{mm})^{-1}\right)$ \\
\hline Granular & $80.23 \pm 18.02^{*}$ & $8.12 \pm 2.32^{*}$ & $4.93 \pm 1.13$ & $0.85 \pm 0.13 *$ & $0.74 \pm 0.11$ \\
Porous & $65.01 \pm 25.54$ & $11.32 \pm 3.34$ & $5.24 \pm 0.64$ & $0.52 \pm 0.09$ & $0.94 \pm 0.15$ \\
\hline
\end{tabular}

i.S: Interception surface, BV: bone volume, TV: tissue volume, BS: bone surface, BS/BV: bone specific surface, BS/TV: bone surface density, Tb.Th: trabecular thickness, Tb.N: trabecular number, Tb.Sp: trabecular separation, ${ }^{*} p<0.05$.

\section{Discussion}

After dental extraction, alveolar ridge maintenance is requisite for healing for bone dimensional changes. The implants in tooth extraction sockets from bone substitute materials have positive effects on bone healing and prevent atrophy in circumferential alveolar bones (14). HA has since long been among the most studied biomaterials in the medical field for both its proven biocompatibility and for being the main constituent of the mineral part of bone. It possesses unique properties, such as the ability to chemically bond to bone, lack of toxicity or inflammation, and ability to stimulate bone growth through a direct action on osteoblasts. HA is widely used in periodontology and in oral and maxillofacial surgery. Its use in oral implantology, is widely practiced and well-established, as this substance has excellent osteoinductive capacity and improves bone-toimplant integration (15). In this study, we evaluated bone regeneration according to $\mathrm{HA}$ manufacturing method in canine alveolar sockets.

Clinicians have been diagnosing, treatment planning, placing and restoring dental implants using radiographs to assess bone anatomy. CT has led to a new era of implant imaging, enabling evaluation of proposed dental implant sites and providing diagnostic information that other imaging or combinations of imaging techniques cannot provide. In the present study, bone regeneration according to HA types was evaluated with CT, micro-CT, and fluorescence microscopic evaluations. CT measurements in our study were carried at 4, 6, and 8 weeks after HA implantation. The 

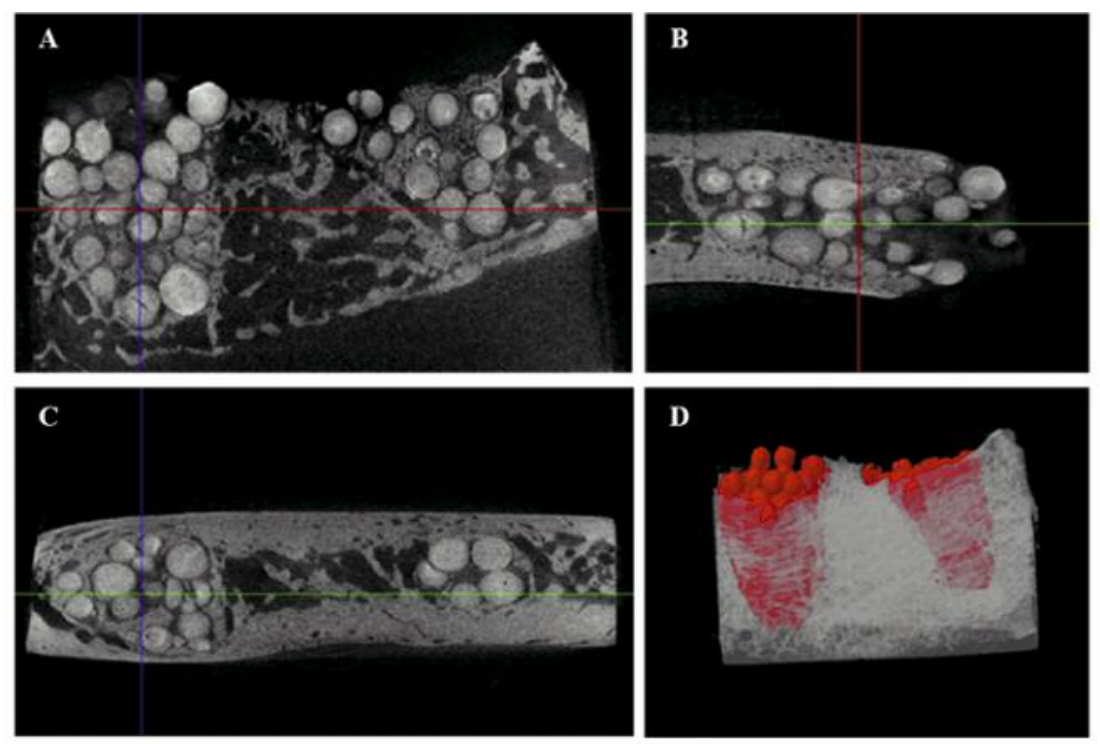

Figure 4. Micro-computed tomography photos of grafting treated with granular hydroxyapatite in canine alveolar socket at 8 weeks postimplantation. Microcomputed tomography in transaxial (A), sagittal (B), coronal $(C)$ axes, and $3 D(D)$.
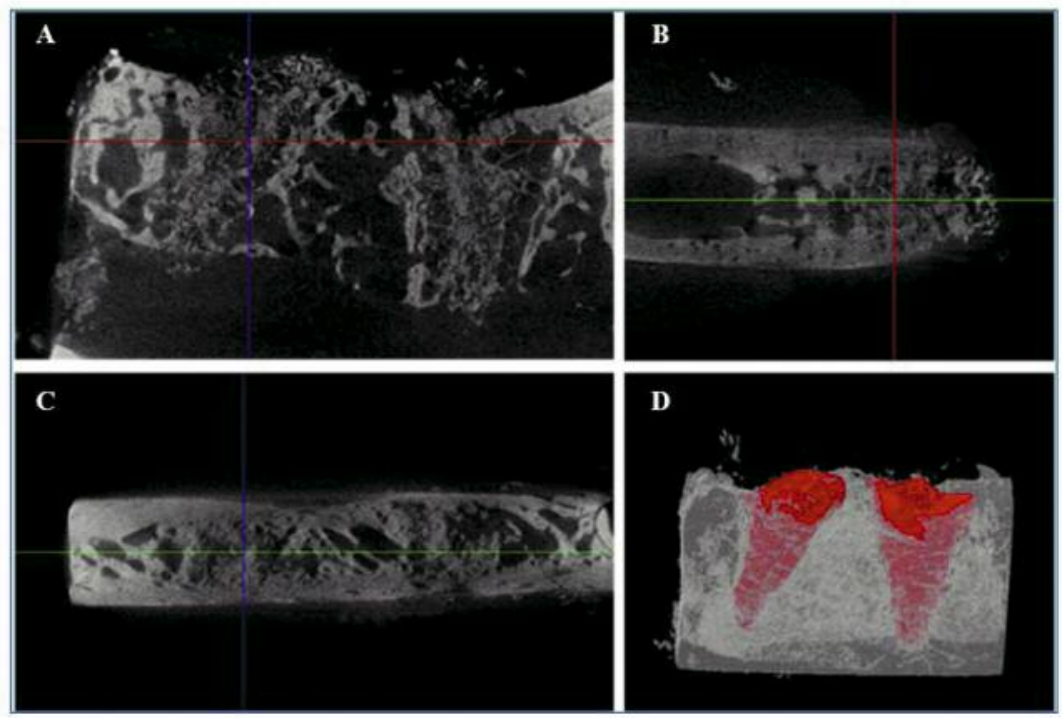

Figure 5. Micro-Ct photos of grafting treated with porous HA scaffold in canine alveolar socket at 8 weeks post-implantation. Micro-computed tomography in transaxial (A), sagittal (B), coronal $(C)$ axes, and $3 D(D)$.

timing was based on animal experiments which indicated that 4 weeks sufficed for angiogenesis and bone formation $(16,17)$. This period is a clinically acceptable standard time for an early evaluation of bone formation (18). This is because the intrinsic density of the granules and particles was as high as that of cortical bone. Bone formation in our study was observed during all experiment periods. The osteogenic effects of HA were maintained well in alveolar sockets, and there was new bone formation in particle spaces. HA materials gave strong results in bone mineralization in the early stage of dental extraction.

In micro-CT parameters, interception surface is the part of the volume-of-interest boundary surface that runs through solid objects. High interception surface values in the gHA and 
4 Weeks

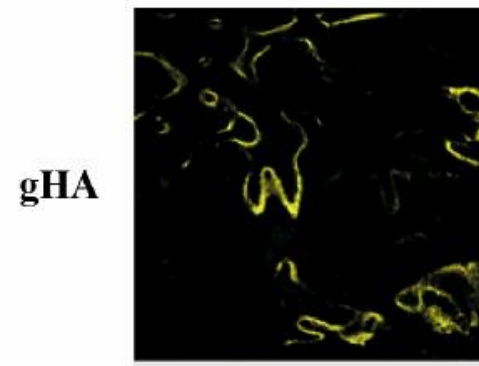

pHA

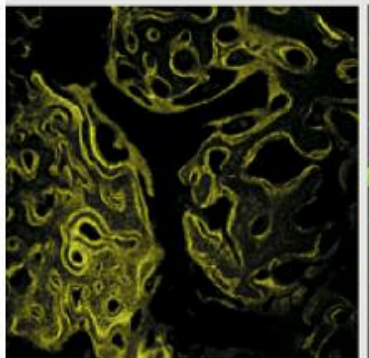

6 Weeks
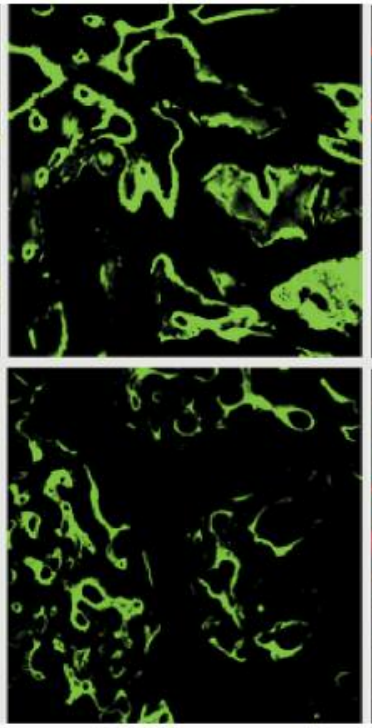

8 Weeks

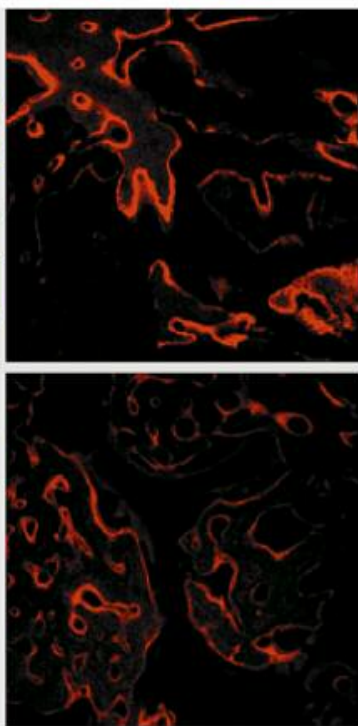

Figure 6. Fluorescence microscopic images of granular hydroxyapatite (gHA) and porous HA (pHA) in canine alveolar sockets at 8 weeks postimplantation. Yellow (oxytetracycline), green (calcein) and red (alizarin) indicated new bone formation after implantation under confocal microscopy.

pHA groups were measured against the control group. Our findings were consistent in that high connectivity between the exterior bone and filling material was good for mechanical stabilization (19). Bone-specific surface represents the characteristics of the structural complexity and is affected by the thickness. Bone-specific surface is at a low value when trabecular thickness and bone surface density/tissue volume are at a high level (20). We thought that the bone-specific surface might be affected by structural changes. The size of HA granules was larger than that of inter-connected pores in the pHA scaffold. From these points, the bone-specific surface may be affected depending on structural changes. Therefore, if the bone parameters by micro-CT are associated with bone loss in osteoporosis, values of $\mathrm{BMD}$, bone volume/tissue volume, trabecular thickness, and trabecular number will decrease, and trabecular separation and bone surface/bone volume will increase $(21,22)$. In this study, trabecular thickness and low trabecular number were higher in the gHA group than in the pHA group. Given these findings, the measurement of BMD and bone volume fraction using CT or micro-CT can be over-estimated depending on the characteristics of bone substitutes. These findings were in accordance with published results claiming that it is hard to estimate osteogenic activity with CT images because of the inherent radio-opacity of particles, granules and porous HA bone grafts of individual bone substitutes (23).

In fluorescence microscopic findings, at 4 and 8 weeks post-implantation the pHA group had more osteocytes compared with the gHA group, and there was new bone formation originating from the alveolar sockets. New bone formation or a significant increase of osteophytes is observed within 2 months in canine alveolar sockets. In the present study, polyurethane template-coating was used to create a HA scaffold with a controllable pore size and good pore interconnectivity. This method creates a friendly structure for cells and tissues that is similar to the natural bone structure. The importance of having interconnected pHA scaffolds was demonstrated in our earlier study (5, 24), indicating successful bone formation, mineralization, and vascularization by 12 weeks post-implantation in a canine defect model.

Our results showed that new bone formation of $\mathrm{pHA}$ scaffold type is faster than that of the gHA form. We found that alveolar sockets in canine mandibles were completely healed when the alveolar socket was filled with HA scaffold containing an isotropic pore structure. Observations from this study, therefore, suggest that the pHA scaffold is suitable for osteogenesis and may be an ideal scaffold for repair of alveolar sockets.

\section{Acknowledgements}

This research was supported by Basic Science Research Program through the National Research Foundation of Korea (NRF) funded by the Ministry of Education (2015R1D1A1A01060583) and the Next-Generation BioGreen 21 Program (PJ01135201), Rural Development Administration, Republic of Korea. 


\section{References}

1 Block MS and Kent JN: A comparison of particulate and solid root forms of hydroxyapatite in dog extraction sites. J Oral Maxillofac Surg 44: 89-93, 1986.

2 Balasundaram G, Sato M and Webster TJ: Using hydroxyapatite nanoparticles and decreased crystallinity to promote osteoblast adhesion similar to functionalizing with RGD. Biomaterials 27: 2798-2805, 2006.

3 Kim JM, Son JS, Kang SS, Kim G and Choi SH: Bone regeneration of hydroxyapatite/alumina bilayered scaffold with $3 \mathrm{~mm}$ passage-like medullary canal in canine tibia model. BioMed Res Int 2015: 235108, 2015.

4 Son JS, Appleford M, Ong JL, Wenke JC, Kim JM, Choi SH and Oh DS: Porous hydroxyapatite scaffold with three-dimensional localized drug delivery system using biodegradable microspheres. J Control Release 153: 133-140, 2011.

5 Son JS, Kim JM, Han M, Choi SH, Lee FY and Oh DS: Bone regeneration of tibial segmental defect using isotropic-pore structures hydroxyapatite/alumina bilayered scaffold: in vivo pilot study. J Long Term Eff Med Implants 21: 159-167, 2011.

6 Chang BS, Lee CK, Hong KS, Youn HJ, Ryu HS, Chung SS and Park KW: Osteoconduction at porous hydroxyapatite with various pore configurations. Biomaterials 21: 1291-1298, 2002.

7 Formica CA, Nieves JW, Cosman F, Garrett P and Lindsay R: Comparative assessment of bone mineral measurements using dual X-ray absorptiometry and peripheral quantitative computed tomography. Osteoporos Int 8: 460-467, 1998.

8 Ebbesen EN, Thomsen JS, Beck-Nielsen H, Nepper-Rasmussen $\mathrm{HJ}$ and Mosekilde L: Lumbar vertebral body compressive strength evaluated by dual-energy X-ray absorptiometry, quantitative computed tomography, and ashing. Bone 25: 713-24, 1999.

9 Schouten C, Meijer GJ, van den Beucken JJ, Spauwen PH and Jansen JA: The quantitative assessment of peri-implant bone responses using histomorphometry and micro-computed tomography. Biomaterials 30: 4539-4549, 2009.

10 Park CH, Abramson ZR, Taba Jr. M, Jin Q, Chang J, Kreider JM, Goldstein SA and Giannobile WV: Three-dimensional microcomputed tomographic imaging of alveolar bone in experimental bone loss or repair. J Periodontol 78: 273-281, 2007.

11 Gielkens PF, Schortinghuis J, de Jong JR, Huysmans MC, Leeuwen MB, Raghoebar GM, Bos RR and Stegenga B: A comparison of micro-CT, microradiography and histomorphometry in bone research. Arch Oral Biol 53: 558-566, 2008.

12 Faulkner KG, Gluer CC, Majumdar S, Lang P, Engelke K and Genant HK: Noninvasive measurements of bone mass, structure, and strength: current methods and experimental techniques. Am J Roentgenol 157: 1229-1237, 1991.

13 Appleford MR, Oh S, Cole JA, Protivinsky J and Ong JL: Ultrasound effect on osteoblast precursor cells in trabecular calcium phosphate scaffolds. Biomaterials 28: 4788-4794, 2007.
14 Rothamel D, Schwarz F, Herten M, Engelhardt E, Donath K, Kuehn $\mathrm{P}$ and Becker J: Dimensional ridge alterations following socket preservation using a nanocrystalline hydroxyapatite paste: a histomorphometrical study in dogs. Int J Oral Maxillofac Surg 37: 741-747, 2008.

15 Pepla E, Besherat LK, Palaia G, Tenore G and Migliau G: Nanohydroxyapatite and its applications in preventive, restorative and regenerative dentistry: a review of literature. Ann Stomatol 3: 108-114, 2014.

16 Schmid J, Wallkamm B, Hammerle CH, Gogolewski S and Lang NP: The significance of angiogenesis in guided bone regeneration. A case report of a rabbit experiment. Clin Oral Implants Res 8: 244-248, 1997.

17 Herron S, Thordarson DB, Winet $\mathrm{H}$, Luk $\mathrm{A}$ and Bao JY: Ingrowth of bone into absorbable bone cement: an in vivo microscopic evaluation. Am J Orthop 32: 581-584, 2003.

18 Mankani MH, Kuznetsov SA, Avila NA, Kingman A and Robey PG: Bone formation in transplants of human bone marrow stromal cells and hydroxyapatite-tricalcium phosphate: prediction with quantitative CT in mice. Radiology 230: 369-376, 2004.

19 Roshan-Ghias A, Terrier A, Bourban PE and Pioletti DP: In vivo cyclic loading as a potent stimulatory signal for bone formation inside tissue engineering scaffold. Eur Cell Mater 19: 41-49, 2010.

20 Kim DG, Christopherson GT, Dong XN, Fyhrie DP and Yeni YN: The effect of microcomputed tomography scanning and reconstruction voxel size on the accuracy of stereological measurements in human cancellous bone. Bone 35: 1375-1382, 2004.

21 Liu YF, Wu LA, Wang J, Wen LY and Wang XJ: Microcomputerized tomography analysis of alveolar bone loss in ligature- and nicotine-induced experimental periodontitis in rats. J Periodontal Res 45: 714-719, 2010.

22 Shimizu M, Furuya R, Kawawa T and Sasaki T: Bone wound healing after maxillary molar extraction in ovariectomized aged rats: quantitative backscattered electron image analysis. Anat Rec 259: 76-85, 2000.

23 Baramki HG, Steffen T, Lander P, Chang M and Marchesi D: The efficacy of interconnected porous hydroxyapatite in achieving posterolateral lumbar fusion in sheep. Spine 25: 1053-1060, 2000.

24 Kim JM, Han TS, Kim MH, Oh DS, Kang SS, Kim G, Kwon TY, Kim KH, Lee KB, Son JS and Choi SH: Osteogenic evaluation of calcium phosphate scaffold with drug-loaded poly (lactic-co-glycolic acid) microspheres in beagle dogs. Tissue Eng Regen Med 9: 175-183, 2012.
Received January 12, 2017

Revised March 6, 2017

Accepted March 10, 2017 